\title{
ASNP: A Tame Fragment of Existential Second-Order Logic
}

\author{
Manuel Bodirsky $\left.{ }^{(}\right)$, Simon Knäuer, and Florian Starke \\ Institute of Algebra, TU Dresden, Dresden, Germany \\ manuel. bodirsky@tu-dresden.de
}

\begin{abstract}
Amalgamation $S N P(A S N P)$ is a fragment of existential second-order logic that strictly contains binary connected MMSNP of Feder and Vardi and binary connected guarded monotone SNP of Bienvenu, ten Cate, Lutz, and Wolter; it is a promising candidate for an expressive subclass of NP that exhibits a complexity dichotomy. We show that ASNP has a complexity dichotomy if and only if the infinitedomain dichotomy conjecture holds for constraint satisfaction problems for first-order reducts of binary finitely bounded homogeneous structures. For such CSPs, powerful universal-algebraic hardness conditions are known that are conjectured to describe the border between NPhard and polynomial-time tractable CSPs. The connection to CSPs also implies that every ASNP sentence can be evaluated in polynomial time on classes of finite structures of bounded treewidth. We show that the syntax of ASNP is decidable. The proof relies on the fact that for classes of finite binary structures given by finitely many forbidden substructures, the amalgamation property is decidable.
\end{abstract}

\section{Introduction}

Feder and Vardi in their groundbreaking work [15] formulated the famous dichotomy conjecture for finite-domain constraint satisfaction problems, which has recently been resolved $[11,26]$. Their motivation to study finite-domain CSPs was the question which fragments of existential second-order logic might exhibit a complexity dichotomy in the sense that every problem that can be expressed in the fragment is either in $\mathrm{P}$ or NP-complete. Existential second-order logic without any restriction is known to capture NP [14] and hence does not have a complexity dichotomy by an old result of Ladner [24]. Feder and Vardi proved that even the fragments of monadic SNP and monotone SNP do not have a complexity dichotomy since every problem in NP is polynomial-time equivalent to a problem that can be expressed in these fragments. However, the dichotomy for finite-domain CSPs implies that monotone monadic SNP (MMSNP) has a dichotomy, too $[15,23]$.

This work has been supported by the European Research Council (ERC) under the European Union's Horizon 2020 research and innovation programme (Grant Agreement No 681988, CSP-Infinity) and by DFG Graduiertenkolleg 1763 (QuantLA).

(C) Springer Nature Switzerland AG 2020

M. Anselmo et al. (Eds.): CiE 2020, LNCS 12098, pp. 149-162, 2020.

https://doi.org/10.1007/978-3-030-51466-2_13 
MMSNP is also known to have a tight connection to a certain class of infinitedomain CSPs [7]: an MMSNP sentence is equivalent to a connected MMSNP sentence if and only if it describes an infinite-domain CSP. Moreover, every problem in MMSNP is equivalent to a finite disjunction of connected MMSNP sentences. The infinite structures that appear in this connection are tame from a model-theoretic perspective: they are reducts of finitely bounded homogeneous structures (see Sect.4.1). CSPs for such structures are believed to have a complexity dichotomy, too; there is even a known hardness condition such that all other CSPs in the class are conjectured to be in $\mathrm{P}[8]$. The hardness condition can be expressed in several equivalent forms $[1,2]$.

In this paper we investigate another candidate for an expressive logic that has a complexity dichotomy. Our minimum requirement for what constitutes a logic is relatively liberal: we require that the syntax of the logic should be decidable. The same requirement has been made for the question whether there exists a logic that captures the class of polynomial-time solvable decision problems (see, e.g., [19,20]). The idea of our logic is to modify monotone SNP so that only CSPs for model-theoretically tame structures can be expressed in the logic; the challenge is to come up with a definition of such a logic which has a decidable syntax. We would like to require that the (universal) first-order part of a monotone SNP sentence describes an amalgamation class. We mention that the Joint Embedding Property (JEP), which follows from the Amalgamation Property $(A P)$, has recently been shown to be undecidable [10]. In contrast, we use the fact that the AP for binary signatures is decidable (Sect.5). We call our new logic Amalgamation SNP (ASNP). This logic contains binary connected MMSNP; it also contains the more expressive logic of binary connected guarded monotone SNP. Guarded monotone SNP (GMSNP) has been introduced in the context of knowledge representation [3] (see Sect.6). We show that ASNP has a complexity dichotomy if and only if the infinite-domain dichotomy conjecture holds for constraint satisfaction problems for first-order reducts of binary finitely bounded homogeneous structures. In particular, every problem that can be expressed in ASNP is a CSP for some countably infinite $\omega$-categorical structure $\mathfrak{B}$. In Sect. 7 we present an example application of this fact: every problem that can be expressed in one of these logics can be solved in polynomial time on instances of bounded treewidth.

\section{Constraint Satisfaction Problems}

Let $\mathfrak{A}, \mathfrak{B}$ be structures with a finite relational signature $\tau$; each symbol $R \in \tau$ is equipped with an arity $\operatorname{ar}(R) \in \mathbb{N}$. A function $h: A \rightarrow B$ is called a homomorphism from $\mathfrak{A}$ to $\mathfrak{B}$ if for every $R \in \tau$ and $\left(a_{1}, \ldots, a_{\operatorname{ar}(R)}\right) \in R^{\mathfrak{A}}$ we have $\left(h\left(a_{1}\right), \ldots, h\left(a_{\operatorname{ar}(R)}\right)\right) \in R^{\mathfrak{B}} ;$ in this case we write $\mathfrak{A} \rightarrow \mathfrak{B}$. We write $\operatorname{CSP}(\mathfrak{B})$ for the class of all finite $\tau$-structures $\mathfrak{A}$ such that $\mathfrak{A} \rightarrow \mathfrak{B}$.

Example 1. If $\mathfrak{B}=K_{3}$ is the 3-clique, i.e., the complete undirected graph with three vertices, then $\operatorname{CSP}(\mathfrak{B})$ is the graph 3 -colouring problem, which is NPcomplete [18]. 
Example 2. If $\mathfrak{B}=(\mathbb{Q} ;<)$ then $\operatorname{CSP}(\mathfrak{B})$ is the digraph acyclicity problem, which is in $\mathrm{P}$.

Example 3. If $\mathfrak{B}=(\mathbb{Q} ;$ Betw $)$ for Betw $:=\{(x, y, z) \mid x<y<z \vee z<y<x\}$ then $\operatorname{CSP}(\mathfrak{B})$ is the Betweenness problem, which is NP-complete [18].

A homomorphism $h$ from $\mathfrak{A}$ to $\mathfrak{B}$ is called an embedding of $\mathfrak{A}$ into $\mathfrak{B}$ if $h$ is injective and for every $R \in \tau$ and $a_{1}, \ldots, a_{\operatorname{ar}(R)} \in A$ we have $\left(a_{1}, \ldots, a_{\operatorname{ar}(R)}\right) \in$ $R^{\mathfrak{A}}$ if and only if $\left(h\left(a_{1}\right), \ldots, h\left(a_{\operatorname{ar}(R)}\right)\right) \in R^{\mathfrak{B}}$; in this case we write $\mathfrak{A} \hookrightarrow \mathfrak{B}$. The union of two $\tau$-structures $\mathfrak{A}, \mathfrak{B}$ is the $\tau$-structure $\mathfrak{A} \cup \mathfrak{B}$ with domain $A \cup B$ and the relation $R^{\mathfrak{A} \cup \mathfrak{B}}:=R^{\mathfrak{A}} \cup R^{\mathfrak{B}}$ for every $R \in \tau$. The intersection $\mathfrak{A} \cap \mathfrak{B}$ is defined analogously. A disjoint union of $\mathfrak{A}$ and $\mathfrak{B}$ is the union of isomorphic copies of $\mathfrak{A}$ and $\mathfrak{B}$ with disjoint domains. As disjoint unions are unique up to isomorphism, we usually speak of the disjoint union of $\mathfrak{A}$ and $\mathfrak{B}$, and denote it by $\mathfrak{A} \uplus \mathfrak{B}$. A structure is connected if it cannot be written as a disjoint union of at least two structures with non-empty domain. A class of structures $\mathcal{C}$ is closed under inverse homomorphisms if whenever $\mathfrak{B} \in \mathcal{C}$ and $\mathfrak{A}$ homomorphically maps to $\mathfrak{B}$ we have $\mathfrak{A} \in \mathcal{C}$. If $\tau$ is a finite relational signature, then it is well-known and easy to see $[5]$ that $\mathcal{C}=\operatorname{CSP}(\mathfrak{B})$ for a countably infinite $\tau$-structure $\mathfrak{B}$ if and only if $\mathcal{C}$ is closed under inverse homomorphisms and disjoint unions.

\section{Monotone SNP}

Let $\tau$ be a finite relational signature, i.e., $\tau$ is a set of relation symbols $R$, each equipped with an arity $\operatorname{ar}(R) \in \mathbb{N}$. An $S N P(\tau-)$ sentence is an existential second-order $(\tau-)$ sentence with a universal first-order part, i.e., a sentence of the form

$$
\exists R_{1}, \ldots, R_{k} \forall x_{1}, \ldots, x_{n}: \phi
$$

where $\phi$ is a quantifier-free formula over the signature $\tau \cup\left\{R_{1}, \ldots, R_{k}\right\}$. We make the additional convention that the equality symbol, which is usually allowed in first-order logic, is not allowed in $\phi$ (see [15]). We write $\llbracket \Phi \rrbracket$ for the class of all finite models of $\Phi$.

Example 4. $\operatorname{CSP}(\mathbb{Q} ;<)=\llbracket \Phi \rrbracket$ for the $\operatorname{SNP}\{<\}$-sentence $\Phi$ given below.

$$
\begin{aligned}
& \exists T \forall x, y, z((\neg(x<y) \vee T(x, y)) \\
&\wedge(\neg T(x, y) \vee \neg T(y, z) \vee T(x, z)) \wedge \neg T(x, x))
\end{aligned}
$$

A class $\mathcal{C}$ of finite $\tau$-structures is said to be in SNP if there exists an SNP $\tau$-sentence $\Phi$ such that $\llbracket \Phi \rrbracket=\mathcal{C}$; we use analogous definitions for all logics considered in this paper. We may assume that the quantifier-free part of SNP sentences is written in conjunctive normal form, and then use the usual terminology (clauses, literals, etc).

Definition 1. An SNP $\tau$-sentence $\Phi$ with quantifier-free part $\phi$ and existentially quantified relation symbols $\sigma$ is called 
- monotone if each literal of $\phi$ with a symbol from $\tau$ is negative, i.e., of the form $\neg R(\bar{x})$ for $R \in \tau$.

- monadic if all the existentially quantified relations are unary.

- connected if each clause of $\phi$ is connected, i.e., the following $\tau \cup \sigma$-structure $\mathfrak{C}$ is connected: the domain of $\mathfrak{C}$ is the set of variables of the clause, and $t \in R^{\mathfrak{C}}$ if and only if $\neg R(t)$ is a disjunct of the clause.

The SNP sentence from Example 4 is monotone, but not monadic, and it can be shown that there does not exist an equivalent MMSNP sentence [4].

Theorem 1 ([5]). Every sentence in connected monotone SNP describes a problem of the form $\operatorname{CSP}(\mathfrak{B})$ for some relational structure $\mathfrak{B}$. Conversely, for every structure $\mathfrak{B}$, if $\operatorname{CSP}(\mathfrak{B})$ is in $S N P$ then it is also in connected monotone SNP.

\section{Amalgamation SNP}

In this section we define the new logic Amalgamation $S N P$ (ASNP). We first revisit some basic concepts from model theory.

\subsection{The Amalgamation Property}

Let $\tau$ be a finite relational signature and let $\mathcal{C}$ be a class of $\tau$-structures. We say that $\mathcal{C}$ is finitely bounded if there exists a finite set of finite $\tau$-structures $\mathcal{F}$ such that $\mathfrak{A} \in \mathcal{C}$ if and only if no structure in $\mathcal{F}$ embeds into $\mathfrak{A}$; in this case we also write $\mathcal{C}=\operatorname{Forb}(\mathfrak{A})$. Note that $\mathcal{C}$ is finitely bounded if and only if there exists a universal $\tau$-sentence $\phi$ (which might involve the equality symbol) such that for every finite $\tau$-structure $\mathfrak{A}$ we have $\mathfrak{A} \models \phi$ if and only if $\mathfrak{A} \in \mathcal{C}$. We say that $\mathcal{C}$ has

- the Joint Embedding Property (JEP) if for all structures $\mathfrak{B}_{1}, \mathfrak{B}_{2} \in \mathcal{C}$ there exists a structure $\mathfrak{C} \in \mathcal{C}$ that embeds both $\mathfrak{B}_{1}$ and $\mathfrak{B}_{2}$.

- the Amalgamation Property $(A P)$ if for any two structures $\mathfrak{B}_{1}, \mathfrak{B}_{2} \in \mathcal{C}$ such that $B_{1} \cap B_{2}$ induce the same substructure in $\mathfrak{B}_{1}$ and in $\mathfrak{B}_{2}$ (a socalled amalgamation diagram) there exists a structure $\mathfrak{C} \in \mathcal{C}$ and embeddings $e_{1}: \mathfrak{B}_{1} \hookrightarrow \mathfrak{C}$ and $e_{2}: \mathfrak{B}_{2} \hookrightarrow \mathfrak{C}$ such that $e_{1}(a)=e_{2}(a)$ for all $a \in B_{1} \cap B_{2}$.

Note that since $\tau$ is relational, the AP implies the JEP. A class of finite $\tau$ structures which has the AP and is closed under induced substructures and isomorphisms is called an amalgamation class.

The age of $\mathfrak{B}$ is the class of all finite $\tau$-structures that embed into $\mathfrak{B}$. We say that $\mathfrak{B}$ is finitely bounded if $\operatorname{Age}(\mathfrak{B})$ is finitely bounded. A relational $\tau$-structure $\mathfrak{B}$ is called homogeneous if every isomorphism between finite substructures of $\mathfrak{B}$ can be extended to an automorphism of $\mathfrak{B}$. Fraïssé's theorem implies that for every amalgamation class $\mathcal{C}$ there exists a countable homogeneous $\tau$-structure $\mathfrak{B}$ with $\operatorname{Age}(\mathfrak{B})=\mathcal{C}$; the structure $\mathfrak{B}$ is unique up to isomorphism, also called the Fraïssé-limit of $\mathcal{C}$. Conversely, it is easy to see that the age of a homogeneous $\tau$ structure is an amalgamation class. A structure $\mathfrak{A}$ is called a reduct of a structure 
$\mathfrak{B}$ if $\mathfrak{A}$ is obtained from $\mathfrak{B}$ by restricting the signature. It is called a first-order reduct of $\mathfrak{B}$ if $\mathfrak{A}$ is obtained from $\mathfrak{B}$ by first expanding by all first-order definable relations, and then restricting the signature. An example of a first-order reduct of $(\mathbb{Q} ;<)$ is the structure $(\mathbb{Q} ;$ Betw) from Example 3 .

\subsection{Defining Amalgamation SNP}

As we have mentioned in the introduction, the idea of our logic is to require that a certain class of finite structures associated to the first-order part of an SNP sentence is an amalgamation class. We then use the fact that for binary signatures, the amalgamation property is decidable (Sect. 5).

Definition 2. Let $\tau$ be a finite relational signature. An Amalgamation SNP $\tau$-sentence is an SNP sentence $\Phi$ of the form $\exists R_{1}, \ldots, R_{k} \forall x_{1}, \ldots, x_{n}$ : $\phi$ where

$-R_{1}, \ldots, R_{k}$ are binary;

- $\phi$ is a conjunction of $\left\{R_{1}, \ldots, R_{k}\right\}$-formulas and of conjuncts of the form $S\left(x_{1}, \ldots, x_{k}\right) \Rightarrow \psi\left(x_{1}, \ldots, x_{k}\right)$ where $S \in \tau$ and $\psi$ is a $\left\{R_{1}, \ldots, R_{k}\right\}$-formula;

- the class of $\left\{R_{1}, \ldots, R_{k}\right\}$-reducts of the finite models of $\phi$ is an amalgamation class.

Note that ASNP inherits from SNP the restriction that equality symbols are not allowed. Also note that Amalgamation SNP sentences are necessarily monotone. This implies in particular that the class of $\left\{R_{1}, \ldots, R_{k}\right\}$-reducts of the finite models of $\phi$ is precisely the class of finite $\left\{R_{1}, \ldots, R_{k}\right\}$-structures that satisfy the conjuncts of $\phi$ that are $\left\{R_{1}, \ldots, R_{k}\right\}$-formulas (i.e., that do not contain any symbol from $\tau$ ).

Example 5. The monotone SNP sentence from Example 4 describing $\operatorname{CSP}(\mathbb{Q} ;<)$ is in ASNP. The problem $\operatorname{CSP}(\mathbb{Q} ;$ Betw $)$ from Example 3 can be expressed by the ASNP sentence

$$
\begin{gathered}
\exists T \forall x, y, z((\operatorname{Betw}(x, y, z) \Rightarrow((T(x, y) \wedge T(y, z)) \vee(T(z, y) \wedge T(y, x))) \\
\wedge((T(x, y) \wedge T(y, z)) \Rightarrow T(x, z)) \wedge \neg T(x, x)) .
\end{gathered}
$$

Note that every finite-domain CSP can be expressed in ASNP; this can be seen similarly as in the argument of Feder and Vardi that finite-domain CSPs can be expressed in MMSNP [15].

Then the class of finite models of the first-order part of $\Phi$ has the JEP, and since equality is not allowed in SNP the class is even closed under disjoint unions; it follows that also $\Phi$ is closed under disjoint unions. It can be shown as in the proof of Theorem 1 that every Amalgamation SNP sentence can be rewritten into an equivalent connected Amalgamation SNP sentence. 


\subsection{ASNP and CSPs}

We present the link between ASNP and infinite-domain CSPs.

Theorem 2. For every ASNP $\tau$-sentence $\Phi$ there exists a first-order reduct $\mathfrak{C}$ of a binary finitely bounded homogeneous structure such that $\operatorname{CSP}(\mathfrak{C})=\llbracket \Phi \rrbracket$.

Proof. Let $\rho$ be the set of existentially quantified relation symbols of $\Phi$. Let $\phi=\forall x_{1}, \ldots, x_{n}: \psi$, for a quantifier-free formula $\psi$ in conjunctive normal form, be the first-order part of $\Phi$. Let $\mathcal{C}$ be the class of $\rho$-reducts of the finite models of $\phi$; by assumption, $\mathcal{C}$ is an amalgamation class. Moreover, $\mathcal{C}$ is finitely bounded because it is the class of models of a universal $\rho$-sentence. Let $\mathfrak{B}$ be the Fraïssélimit of $\mathcal{C}$; then $\mathfrak{B}$ is a finitely bounded homogeneous structure. Let $\mathfrak{C}$ be the $\tau$-structure which is the first-order reduct of the structure $\mathfrak{B}$ where the relation $S^{\mathfrak{C}}$ for $S \in \tau$ is defined as follows: if $\phi_{1}, \ldots, \phi_{s}$ are all the $\rho$-formulas such that $\psi$ contains the conjunct $S\left(x_{1}, \ldots, x_{k}\right) \Rightarrow \phi_{i}\left(x_{1}, \ldots, x_{k}\right)$ for all $i \in\{1, \ldots, s\}$, then the first-order definition of $S$ is given by $S\left(x_{1}, \ldots, x_{k}\right) \Leftrightarrow\left(\phi_{1} \wedge \cdots \wedge \phi_{s}\right)$.

Claim 1. If $\mathfrak{A}$ is a finite $\tau$-structure such that $\mathfrak{A} \rightarrow \mathfrak{C}$, then $\mathfrak{A} \models \Phi$.

Let $h: \mathfrak{A} \rightarrow \mathfrak{C}$ be a homomorphism. Let $\mathfrak{A}^{\prime}$ be the $(\tau \cup \rho)$-expansion of $\mathfrak{A}$ where $R \in \rho$ of arity $l$ denotes $\left\{\left(a_{1}, \ldots, a_{l}\right) \mid\left(h\left(a_{1}\right), \ldots, h\left(a_{l}\right)\right) \in R^{\mathfrak{B}}\right\}$. Then $\mathfrak{A}^{\prime}$ satisfies $\phi$ : to see this, let $a_{1}, \ldots, a_{n} \in A$ and let $\psi^{\prime}$ be a conjunct of $\psi$. Since $\mathfrak{C}=$ $\forall x_{1}, \ldots, x_{n}: \psi$ we have in particular that $\mathfrak{C} \models \psi^{\prime}\left(h\left(a_{1}\right), \ldots, h\left(a_{n}\right)\right)$ and so there must be a disjunct $\psi^{\prime \prime}$ of $\psi^{\prime}$ such that $\mathfrak{C} \models \psi^{\prime \prime}\left(h\left(a_{1}\right), \ldots, h\left(a_{n}\right)\right)$. Then one of the following cases applies.

- $\psi^{\prime \prime}$ is a $\tau$-literal and hence must be negative since $\Phi$ is a monotone SNP sentence. In this case $\mathfrak{C} \models \psi^{\prime \prime}\left(h\left(a_{1}\right), \ldots, h\left(a_{n}\right)\right)$ implies $\mathfrak{A}^{\prime} \models \psi^{\prime \prime}\left(a_{1}, \ldots, a_{n}\right)$ since $h$ is a homomorphism.

- $\psi^{\prime \prime}$ is a $\rho$-literal. Then by the definition of $\mathfrak{A}^{\prime}$ we have that $\mathfrak{A}^{\prime} \models \psi^{\prime \prime}\left(a_{1}, \ldots, a_{n}\right)$ if and only if $\mathfrak{C} \models \psi^{\prime \prime}\left(h\left(a_{1}\right), \ldots, h\left(a_{n}\right)\right)$.

Hence, $\mathfrak{A}^{\prime} \models \psi^{\prime}\left(a_{1}, \ldots, a_{n}\right)$. Since the conjunct $\psi^{\prime}$ of $\psi$ and $a_{1}, \ldots, a_{n} \in A$ were arbitrarily chosen, we have that $\mathfrak{A}^{\prime} \models \forall x_{1}, \ldots, x_{n}$ : $\psi$. Hence, $\mathfrak{A}$ satisfies $\Phi$.

Claim 2. If $\mathfrak{A}$ is a finite $\tau$-structure such that $\mathfrak{A}=\Phi$, then $\mathfrak{A} \rightarrow \mathfrak{C}$.

If $\mathfrak{A}$ has a $(\tau \cup \rho)$-expansion $\mathfrak{A}^{\prime}$ that satisfies $\phi$, then there exists an embedding from the $\rho$-reduct $\mathfrak{A}^{\prime \prime}$ of $\mathfrak{A}^{\prime}$ into $\mathfrak{B}$ by the definition of $\mathfrak{B}$. This embedding is in particular a homomorphism from $\mathfrak{A}$ to $\mathfrak{C}$.

Theorem 3. Let $\mathfrak{C}$ be a first-order reduct of a binary finitely bounded homogeneous structure $\mathfrak{B}$. Then $\operatorname{CSP}(\mathfrak{C})$ can be expressed in ASNP. 
Proof. Let $\sigma$ be the signature of $\mathfrak{B}$ and $\tau$ the signature of $\mathfrak{C}$. We may assume without loss of generality that $\mathfrak{B}$ contains a binary relation $E$ that denotes the equality relation; it is easy to see that an expansion by the equality relation preserves finite boundedness. Consider the structure $\mathfrak{B}^{*}$ with the domain $B \times \mathbb{N}$ where

$$
R^{\mathfrak{B}^{*}}:=\left\{\left(\left(b_{1}, n_{1}\right), \ldots,\left(b_{k}, n_{k}\right)\right) \mid n_{1}, \ldots, n_{k} \in \mathbb{N},\left(b_{1}, \ldots, b_{k}\right) \in R^{\mathfrak{B}}\right\} .
$$

To show that $\mathfrak{B}^{*}$ is homogeneous, let $h$ be an isomorphism between finite substructures of $\mathfrak{B}^{*}$. Let $T \subseteq B$ be the set of all first entries of elements of the first structure. Define $g: T \rightarrow B$ by picking for $b \in T$ an element of the form $(b, n) \in S$ and defining by $g(b):=h(b, n)_{1}$. This is well-defined: if $h$ is defined on $\left(b, n_{1}\right)$ and on $\left(b, n_{2}\right)$, then $\left(\left(b, n_{1}\right),\left(b, n_{2}\right)\right) \in E^{\mathfrak{B}^{*}}$, and hence $h\left(b, n_{1}\right)_{1}=h\left(b, n_{2}\right)_{1}$. The same consideration for $h^{-1}$ shows that $g$ is a bijection, and in fact an isomorphism between finite substructures of $\mathfrak{B}$. By the homogeneity of $\mathfrak{B}$ there exists an extension $g^{*} \in \operatorname{Aut}(\mathfrak{B})$ of $g$. For each $b \in B$ pick a permutation $f_{b}$ of $\mathbb{N}$ that extends the bijection given by $n \mapsto h(b, n)_{2}$. Then the map $h^{*}: B^{*} \rightarrow B^{*}$ given by $h(b, n):=\left(g^{*}(b), f_{b}(n)\right)$ is an automorphism of $\mathfrak{B}^{*}$ that extends $h$. Since $\mathfrak{B}$ is finitely bounded, there exists a universal $\sigma$-formula $\phi$ such that $\operatorname{Age}(\mathfrak{B})=\llbracket \phi \rrbracket$. Note that $\phi$ might contain the equality symbol (which we do not allow in SNP sentences). Let $\phi^{*}$ be the formula obtained from $\phi$ by

- replacing each occurrence of the equality symbol by the symbol $E \in \sigma$;

- joining conjuncts that imply that $E$ denotes an equivalence relation;

- joining for every $R \in \sigma$ of arity $n$ the conjunct

$$
\forall x_{1}, \ldots, x_{n}, y_{1}, \ldots, y_{n}\left(R\left(x_{1}, \ldots, x_{n}\right) \vee \neg R\left(y_{1}, \ldots, y_{n}\right) \vee \bigvee_{i \leq n} \neg E\left(x_{i}, y_{i}\right)\right)
$$

(implementing indiscernibility of identicals for the relation $E$ ).

We claim that $\operatorname{Age}\left(\mathfrak{B}^{*}\right)=\llbracket \phi^{*} \rrbracket$. To see this, let $\mathfrak{A}^{*}$ be a finite $\sigma$-structure. If $\mathfrak{A}^{*}$ satisfies $\phi^{*}$, then every induced substructure $\mathfrak{A}$ of $\mathfrak{A}^{*}$ with the property that $(x, y) \in E^{\mathfrak{A}}$ implies that at most one of $x$ and $y$ is an element of $A$, satisfies $\phi$, and hence is a substructure of $\mathfrak{B}$. This in turn means that $\mathfrak{A}^{*}$ is in $\operatorname{Age}\left(\mathfrak{B}^{*}\right)$. The implications in this statement can be reversed which shows the claim.

Let $\phi^{\prime}$ be the formula obtained from $\phi^{*}$ as follows. For each $S \in \tau$ let $\chi_{S}$ be the first-order definition of $S^{\mathfrak{C}}$ in $\mathfrak{B}$; since $\mathfrak{B}$ is homogeneous we may assume that $\chi_{S}$ is quantifier-free [21]. Furthermore, we may assume that $\chi_{S}$ is given in conjunctive normal form. Let $k$ be the arity of $S$. We then add for each conjunct $\chi_{S}^{\prime}$ of $\chi_{S}$ the conjunct

$$
\forall x_{1}, \ldots, x_{k}\left(S\left(x_{1}, \ldots, x_{k}\right) \Rightarrow \chi_{S}^{\prime}\left(x_{1}, \ldots, x_{k}\right)\right)
$$

By construction, the sentence $\Phi$ obtained from $\phi^{\prime}$ by quantifying all relation symbols of $\sigma$ is an ASNP $\tau$-sentence.

Corollary 1. ASNP has a complexity dichotomy if and only if the infinitedomain dichotomy conjecture is true for first-order reducts of binary finitely bounded homogeneous structures. 


\section{Deciding Amalgamation}

In this section we show how to algorithmically decide whether a given existential second-order sentence is in ASNP. The following is a known fact in the model theory of homogeneous structures (the first author has learned the fact from Gregory Cherlin), but we are not aware of any published proof in the literature.

Theorem 4. Let $\mathcal{F}$ be a finite set of finite binary relational $\tau$-structures. There is an algorithm that decides whether $\operatorname{Forb}(\mathcal{F})$ has the amalgamation property.

Proof. Let $m$ be the maximal size of a structure in $\mathcal{F}$, and let $\ell$ be the number of isomorphism types of two-element structures in $\mathcal{C}:=\operatorname{Forb}(\mathcal{F})$. It is well-known and easy to prove that $\mathcal{C}$ has the amalgamation property if and only if it has the so-called 1-point amalgamation property, i.e., the amalgamation property restricted to diagrams $\left(\mathfrak{B}_{1}, \mathfrak{B}_{2}\right)$ where $\left|B_{1}\right|=\left|B_{2}\right|=\left|B_{1} \cap B_{2}\right|+1$. Suppose that $\left(\mathfrak{B}_{1}, \mathfrak{B}_{2}\right)$ is such an amalgamation diagram without amalgam. Let $B_{0}:=B_{1} \cap B_{2}$. Let $B_{1} \backslash B_{0}=\{p\}$ and $B_{2} \backslash B_{0}=\{q\}$. Let $\mathfrak{D}$ be a $\tau$-structure $\mathfrak{D}$ with domain $B_{1} \cup B_{2}$ such that $\mathfrak{B}_{1}$ and $\mathfrak{B}_{2}$ are substructures of $\mathfrak{D}$. Since $\mathfrak{D}$ by assumption is not an amalgam for $\left(\mathfrak{B}_{1}, \mathfrak{B}_{2}\right)$, there must exist $A=\left\{a_{1}, \ldots, a_{m-2}\right\} \in B_{0}$ such that the substructure of $\mathfrak{D}$ induced by $\left\{a_{1}, \ldots, a_{m-2}, p, q\right\}$ embeds a structure from $\mathcal{F}$.

Note that the number of such $\tau$-structures $\mathfrak{D}$ is bounded by $\ell$ since they only differ by the substructure induced by $p$ and $q$. So let $A_{1}, \ldots, A_{\ell} \subseteq B_{0}$ be a list of sets witnessing that all of these structures $\mathfrak{D}$ embed a structure from $\mathcal{F}$. Let $\mathfrak{C}_{1}$ be the substructure of $\mathfrak{B}_{1}$ induced by $\{p\} \cup A_{1} \cup \cdots \cup A_{\ell}$ and $\mathfrak{C}_{2}$ be the substructure of $\mathfrak{B}_{2}$ induced by $\{q\} \cup A_{1} \cup \cdots \cup A_{\ell}$. Suppose for contradiction that $\left(\mathfrak{C}_{1}, \mathfrak{C}_{2}\right)$ has an amalgam $\mathfrak{C}$; we may assume that this amalgam is of size at most $(m-2) \cdot \ell$. Depending on the two-element structure induced by $\{p, q\}$ in $\mathfrak{C}$, there exists an $i \leq \ell$ such that the structure induced by $\{p, q\} \cup A_{i}$ in $\mathfrak{C}$ embeds a structure from $\mathcal{F}$, a contradiction.

Corollary 2. There is an algorithm that decides for a given existential secondorder sentence $\Phi$ whether it is in ASNP.

Proof. Let $k$ be the maximal number of variables per clause in the first-order part $\phi$ of $\Phi$, and let $\mathcal{F}$ be the set of all structures at most the elements $\{1, \ldots, k\}$ that do not satisfy $\phi$. Then $\operatorname{Forb}(\mathcal{F})=\llbracket \phi \rrbracket$ and the result follows from Theorem 2 .

\section{Guarded Monotone SNP}

In this section we revisit an expressive generalisation of MMSNP introduced by Bienvenu, ten Cate, Lutz, and Wolter [3] in the context of ontology-based data access, called guarded monotone SNP (GMSNP). It is equally expressive as the logic $\mathrm{MMSNP}_{2}$ introduced by Madelaine $[25]^{1}$. We will see that every GMSNP sentence is equivalent to a finite disjunction of connected GMSNP sentences (Proposition 1), each of which lies in ASNP if the signature is binary (Theorem 5).

\footnotetext{
${ }^{1} \mathrm{MMSNP}_{2}$ relates to MMSNP as Courcelle's $\mathrm{MSO}_{2}$ relates to MSO [13].
} 
Definition 3. A monotone $S N P \tau$-sentence $\Phi$ with existentially quantified relations $\rho$ is called guarded if each conjunct of $\Phi$ can be written in the form

$$
\alpha_{1} \wedge \cdots \wedge \alpha_{n} \Rightarrow \beta_{1} \vee \cdots \vee \beta_{m}, \quad \text { where }
$$

- $\alpha_{1}, \ldots, \alpha_{n}$ are atomic $(\tau \cup \rho)$-formulas, called body atoms,

- $\beta_{1}, \ldots, \beta_{m}$ are atomic $\rho$-formulas, called head atoms,

- for every head atom $\beta_{i}$ there is a body atom $\alpha_{j}$ such that $\alpha_{j}$ contains all variables from $\beta_{i}$ (such clauses are called guarded).

We do allow the case that $m=0$, i.e., the case where the head consists of the empty disjunction, which is equivalent to $\perp$ (false).

The next proposition extends a well-known fact for MMSNP to guarded SNP.

Proposition 1. Every GMSNP sentence $\Phi$ is equivalent to a finite disjunction $\Phi_{1} \vee \cdots \vee \Phi_{k}$ of connected GMSNP sentences.

Proof. We prove Proposition 1. Let $\Phi$ be a guarded SNP sentence. Suppose that the quantifier-free part of $\Phi$ has a disconnected clause $\psi$ (Definition 1 ). By definition the variable set can be partitioned into non-empty variable sets $X_{1}$ and $X_{2}$ such that for every negative literal $\neg R\left(x_{1}, \ldots, x_{r}\right)$ of the clause either $\left\{x_{1}, \ldots, x_{r}\right\} \subseteq X_{1}$ or $\left\{x_{1}, \ldots, x_{r}\right\} \subseteq X_{2}$. The same is true for every positive literal, since otherwise the definition of guarded clauses would imply a negative literal on a set that contains $\left\{x_{1}, \ldots, x_{r}\right\}$, contradicting the property above. Hence, $\psi$ can be written as $\psi_{1}(\bar{x}) \vee \psi_{2}(\bar{y})$ for non-empty disjoint tuples of variables $\bar{x}$ and $\bar{y}$. Let $\phi_{1}$ be the formula obtained from $\phi$ by replacing $\psi$ by $\psi_{1}$, and let $\phi_{2}$ be the formula obtained from $\phi$ by replacing $\psi$ by $\psi_{2}$.

Let $P_{1}, \ldots, P_{k}$ be the existential predicates in $\Phi$, and let $\tau$ be the input signature of $\Phi$. It suffices to show that for every $\left(\tau \cup\left\{P_{1}, \ldots, P_{k}\right\}\right)$-expansion $\mathfrak{A}^{\prime}$ of $\mathfrak{A}$ we have that $\mathfrak{A}^{\prime}$ satisfies $\phi$ if and only if $\mathfrak{A}^{\prime}$ satisfies $\phi_{1}$ or $\phi_{2}$. If $\mathfrak{A}^{\prime}$ falsifies a clause of $\phi$, there is nothing to show since then $\mathfrak{A}^{\prime}$ satisfies neither $\phi_{1}$ nor $\phi_{2}$. If $\mathfrak{A}^{\prime}$ satisfies all clauses of $\phi$, it in particular satisfies a literal from $\psi$; depending on whether this literal lies in $\psi_{1}$ or in $\psi_{2}$, we obtain that $\mathfrak{A}^{\prime}$ satisfies $\psi_{1}$ or $\psi_{2}$, and hence $\phi_{1}$ or $\phi_{2}$. Iterating this process for each disconnected clause of $\phi$, we eventually arrive at a finite disjunction of connected guarded SNP sentences.

It is well-known and easy to see [17] that each of $\Phi_{1}, \ldots, \Phi_{k}$ can be reduced to $\Phi$ in polynomial time. Conversely, if each of $\Phi_{1}, \ldots, \Phi_{k}$ is in $\mathrm{P}$, then $\Phi$ is in $\mathrm{P}$, too. It follows in particular that if connected GMSNP has a complexity dichotomy into $\mathrm{P}$ and NP-complete, then so has GMSNP.

Theorem 5. For every sentence $\Phi$ in connected GMSNP there exists a reduct $\mathfrak{C}$ of a finitely bounded homogeneous structures such that $\llbracket \Phi \rrbracket=\operatorname{CSP}(\mathfrak{C})$. If all existentially quantified relation symbols in $\Phi$ are binary then it is equivalent to an ASNP sentence. 
In the proof of Theorem 5 we use a result of Cherlin, Shelah, and Shi [12] in a strengthened form due to Hubička and Nešetřil [22], namely that for every finite set $\mathcal{F}$ of finite $\sigma$-structures, for some finite relational signature $\sigma$, there exists a finitely bounded homogeneous $(\sigma \cup \rho)$-structure $\mathfrak{B}$ such that a finite $\sigma$-structure $\mathfrak{A}$ homomorphically maps to $\mathfrak{B}$ if none of the structures in $\mathcal{F}$ homomorphically maps to $\mathfrak{B}$. We now prove Theorem 5 .

Proof. Let $\Phi$ be a $\tau$-sentence in connected guarded monotone SNP with existentially quantified relation symbols $\left\{E_{1}, \ldots, E_{k}\right\}$. Let $\sigma$ be the signature which contains for every relation symbol $R \in\left\{E_{1}, \ldots, E_{k}\right\}$ two new relation symbols $R^{+}$and $R^{-}$of the same arity and for every relation symbol $R \in \tau$ a new relation symbol $R^{\prime}$. Let $\phi$ be the first-order part of $\Phi$, written in conjunctive normal form, and let $n$ be the number of variables in the largest clause of $\phi$. Let $\phi^{\prime}$ be the sentence obtained from $\phi$ by replacing each occurrence of $R \in\left\{E_{1}, \ldots, E_{k}\right\}$ by $R^{+}$and each occurrence of $\neg R$ by $R^{-}$, and finally each occurrence of $R \in \tau$ by $R^{\prime}$. Let $\mathcal{F}$ be the (finite) class of all finite $\sigma$-structures with at most $n$ elements that do not satisfy $\phi^{\prime}$. We apply the mentioned theorem of Hubička and Nešetřil to $\mathcal{F}$, and obtain a finitely bounded homogeneous $\sigma \cup \rho$-structure $\mathfrak{B}$ such that the age of the $\sigma$-reduct $\mathfrak{C}$ of $\mathfrak{B}$ equals $\operatorname{Forb}(\mathcal{N})$. We say that $S \subseteq B$ is correctly labelled if for every $R \in\left\{E_{1}, \ldots, E_{k}\right\}$ of arity $m$ and $s_{1}, \ldots, s_{m} \in S$ we have $R^{-}\left(s_{1}, \ldots, s_{m}\right)$ if and only if $\neg R\left(s_{1}, \ldots, s_{m}\right)$. Let $\mathfrak{B}^{\prime}$ the $\tau \cup \sigma \cup \rho$-expansion of $\mathfrak{B}$ where $R \in \tau$ of arity $m$ denotes

$$
\left\{\left(t_{1}, \ldots, t_{m}\right) \in\left(R^{\prime}\right)^{\mathfrak{B}} \mid\left\{t_{1}, \ldots, t_{m}\right\} \text { is correctly labelled }\right\} .
$$

Since $\mathfrak{B}$ is finitely bounded homogeneous, $\mathfrak{B}^{\prime}$ is finitely bounded homogeneous, too. Let $\mathfrak{C}$ be the $\tau$-reduct of $\mathfrak{B}^{\prime}$. We claim that $\llbracket \Phi \rrbracket=\operatorname{CSP}(\mathfrak{C})$. First suppose that $\mathfrak{A}$ is a finite $\tau$-structure that satisfies $\Phi$. Then it has an $\left\{E_{1}, \ldots, E_{k}\right\}$-expansion $\mathfrak{A}^{\prime}$ that satisfies $\phi$. Let $\mathfrak{A}^{\prime \prime}$ be the $\sigma$-structure with the same domain as $\mathfrak{A}^{\prime}$ where

- $R^{\prime}$ denotes $R^{\mathfrak{A}^{\prime}}$ for each $R \in \tau$;

- $R^{+}$denotes $R^{\mathfrak{A}^{\prime}}$ for each $R \in\left\{E_{1}, \ldots, E_{k}\right\}$;

- $R^{-}$denotes $\neg R^{\mathfrak{A}^{\prime}}$ for each $R \in\left\{E_{1}, \ldots, E_{k}\right\}$.

Then $\mathfrak{A}^{\prime \prime}$ satisfies $\phi^{\prime}$, and hence embeds into $\mathfrak{B}$. This embedding is a homomorphism from $\mathfrak{A}$ to $\mathfrak{C}$ since the image of the embedding is correctly labelled by the construction of $\mathfrak{A}^{\prime \prime}$.

Conversely, suppose that $\mathfrak{A}$ has a homomorphism $h$ to $\mathfrak{C}$. Let $\mathfrak{A}^{\prime}$ be the $\tau \cup\left\{E_{1}, \ldots, E_{k}\right\}$-expansion of $\mathfrak{A}$ by defining $\left(a_{1}, \ldots, a_{n}\right) \in R^{\mathfrak{A}}$ if and only if $\left(h\left(a_{1}\right), \ldots, h\left(a_{n}\right)\right) \in R^{\mathfrak{B}^{\prime}}$, for every $n$-ary $R \in\left\{E_{1}, \ldots, E_{k}\right\}$. Then each clause of $\phi$ is satisfied, because each clause of $\phi$ is guarded: let $x_{1}, \ldots, x_{m}$ be the variables of some clause of $\phi$. If $a_{1}, \ldots, a_{m} \in A$ satisfy the body of this clause, and $\psi\left(a_{i_{1}}, \ldots, a_{i_{l}}\right)$ is a head atom of such a clause, then the set $\left\{h\left(a_{i_{1}}\right), \ldots, h\left(a_{i_{l}}\right)\right\}$ is correctly labelled. This implies that some of the head atoms of the clause must be true in $\mathfrak{A}^{\prime}$ because $\mathfrak{B}^{\prime}$ satisfies $\phi^{\prime}$. The second statement follows from Theorem 3 .

The following example shows that GMSNP does not contain ASNP. 
Example 6. $\operatorname{CSP}(\mathbb{Q} ;<$ ) is in ASNP (see Example 5) but not in GMSNP. Indeed, suppose that $\Phi$ is a GMSNP sentence which is true on all finite directed paths. We assume that the quantifier-free part $\phi$ of $\Phi$ is in conjunctive normal form. Let $\rho$ be the existentially quantified relation symbols of $\Phi$, let $k:=|\rho|$, and let $l$ be the number of variables in $\Phi$. Every directed path, viewed as a $\{<\}$-structure, satisfies $\Phi$, and therefore has an $\{<\} \cup \rho$-expansion $\mathfrak{A}$ that satisfies $\phi$. Note that there are finitely many different $\{<\} \cup \rho$-expansions of a path of length $l \in \mathbb{N}$; let $p \in \mathbb{N}$ be this number. Hence, for a path of length $L:=(p+1) l$, there must be $i, j \in\{0, \ldots, p\}$ with $i<j$ such that the substructures of $\mathfrak{A}$ induced by $i l+1, i l+2, \ldots, i l+l$ and by $j l+1, j l+2, \ldots, j l+l$ are isomorphic. We then claim that the directed cycle $(i+1) l+1,(i+1) l+2, \ldots, j l+1, \ldots, j l+l,(i+1) l+1$ satisfies $\Phi$ : this is witnessed by the $\{<\} \cup \rho$-expansion inherited from $\mathfrak{A}$ which satisfies $\phi$ since each clause in $\phi$ is guarded. Hence, $\Phi$ does not express digraph acyclicity.

\section{Application: Instances of Bounded Treewidth}

If a computational problem can be formulated in ASNP or in GMSNP, then this has remarkable consequences besides a potential complexity dichotomy. In this section we show that every problem that can be formulated in ASNP or in GMSNP is in $\mathrm{P}$ when restricted to instances of bounded treewidth. The corresponding result for Monadic Second-Order Logic (MSO) instead of ASNP is a famous theorem of Courcelle [13]. We strongly believe that ASNP is not contained in MSO (consider for instance the Betweenness Problem from Example 3), so our result appears to be incomparable to Courcelle's.

In the proof of our result, we need the following concepts from model theory. A first-order theory $T$ is called $\omega$-categorical if all countable models of $T$ are isomorphic [21]. A structure $\mathfrak{B}$ is called $\omega$-categorical if its first-order theory (i.e., the set of first-order sentences that hold in $\mathfrak{B}$ ) is $\omega$-categorical. Note that with this definition, finite structures are $\omega$-categorical. Another classic example is the structure $(\mathbb{Q} ;<)$. The definition of treewidth can be treated as a black box in our proof, and we refer the reader to [6].

Theorem 6. Let $\Phi$ be an ASNP or a connected GMSNP $\tau$-sentence and let $k \in$ $\mathbb{N}$. Then the problem to decide whether a given finite $\tau$-structure $\mathfrak{A}$ of treewidth at most $k$ satisfies $\Phi$ can be decided in polynomial time with a Datalog program (of width $k$ ).

Proof. Since structures that are homogeneous in a finite relational language are $\omega$-categorical [21] and first-order reducts of $\omega$-categorical structures are $\omega$-categorical [21], Theorem 2 and Theorem 5 imply that the problem to decide whether a finite $\tau$-structure satisfies $\phi$ can be formulated as $\operatorname{CSP}(\mathfrak{B})$ for an $\omega$-categorical structure $\mathfrak{B}$. Then the statement follows from Corollary 1 in [6].

Remark 1. In Theorem 6 it actually suffices to assume that the core of $\mathfrak{A}$ has treewidth at most $k$. 
Corollary 3. Let $\Phi$ be a GMSNP $\tau$-sentence and let $k \in \mathbb{N}$. Then there is a polynomial-time algorithm that decides whether a given $\tau$-structure of treewidth at most $k$ satisfies $\Phi$.

Proof. Immediate from Theorem 1 and Theorem 6.

\section{Conclusion and Open Problems}

ASNP is a candidate for an expressive logic with a complexity dichotomy: every problem in ASNP is NP-complete or in $\mathrm{P}$ if and only if the infinite-domain dichotomy conjecture for first-order reducts of binary finitely bounded homogeneous structures holds. See Fig. 1 for the relation to other candidate logics that are known to have a dichotomy, might have a complexity, or provably do not have a dichotomy.

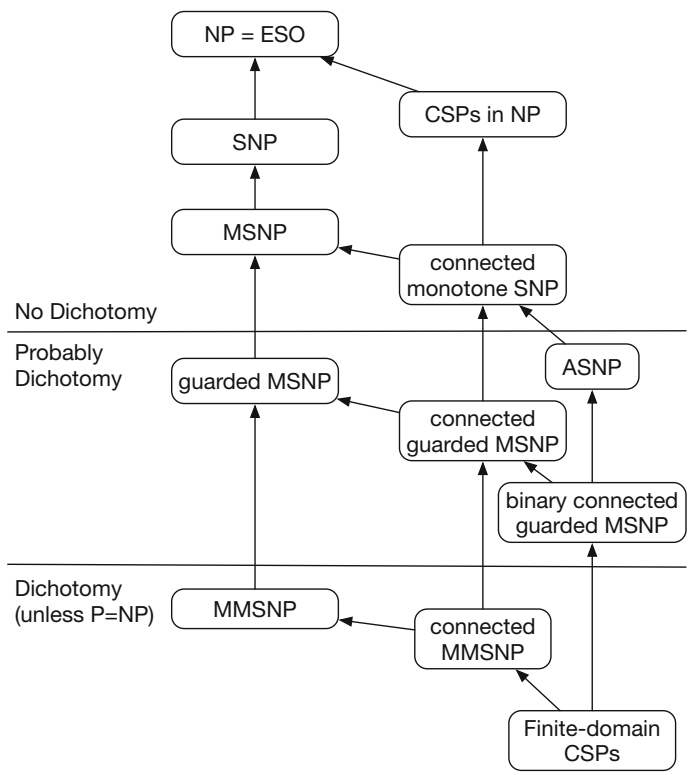

Fig. 1. Fragments of existential second-order logic and complexity dichotomies.

We presented an application of ASNP concerning the evaluation of computational problems on classes of structures of bounded treewidth. We also proved that the syntax of ASNP is algorithmically decidable. The following problems concerning ASNP are open.

1. Is the Amalgamation Property decidable for (not necessarily binary) classes given by finitely many forbidden substructures?

2. Is every binary CSP in Monadic Second-Order Logic (MSO) also in ASNP? 
3. Is every problem in NP polynomial-time equivalent to a problem in Amalgamation SNP if we drop the monotonicity assumption?

4. Is there a natural logic (which in particular has an effective syntax) that contains both ASNP and connected GMSNP and which describes CSPs for reducts of finitely bounded homogeneous structures?

\section{References}

1. Barto, L., Kompatscher, M., Olšák, M., Pham, T.V., Pinsker, M.: Equations in oligomorphic clones and the constraint satisfaction problem for $\omega$-categorical structures. J. Math. Logic, 19(2), \#1950010 (2019). An extended abstract appeared at the Proceedings of the 32nd Annual ACM/IEEE Symposium on Logic in Computer Science - LICS'17

2. Barto, L., Pinsker, M.: The algebraic dichotomy conjecture for infinite domain constraint satisfaction problems. In: Proceedings of the 31th Annual IEEE Symposium on Logic in Computer Science, LICS 2016, pp. 615-622 (2016). Preprint arXiv: 1602.04353

3. Bienvenu, M., ten Cate, B., Lutz, C., Wolter, F.: Ontology-based data access: a study through disjunctive datalog, CSP, and MMSNP. ACM Trans. Database Syst. 39(4), 33 (2014)

4. Bodirsky, M.: Constraint satisfaction with infinite domains. Dissertation, Humboldt-Universität zu Berlin (2004)

5. Bodirsky, M.: Complexity classification in infinite-domain constraint satisfaction. Mémoire d'habilitation à diriger des recherches, Université Diderot - Paris 7. arXiv:1201.0856 (2012)

6. Bodirsky, M., Dalmau, V.: Datalog and constraint satisfaction with infinite templates. J. Comput. Syst. Sci. 79, 79-100 (2013). A preliminary version appeared in the proceedings of the Symposium on Theoretical Aspects of Computer Science (STACS'05)

7. Bodirsky, M., Madelaine, F., Mottet, A.: A universal-algebraic proof of the complexity dichotomy for Monotone Monadic SNP. In: Proceedings of the Symposium on Logic in Computer Science (LICS), pp. 105-114 (2018). Preprint available under arXiv: 1802.03255

8. Bodirsky, M., Pinsker, M., Pongrácz, A.: Projective clone homomorphisms. J. Symb. Logic (2014). Preprint arXiv:1409.4601

9. Bodirsky, M., Rydval, J.: Temporal constraint satisfaction problems in fixed point logic (2019). Preprint

10. Braunfeld, S.: Towards the undecidability of atomicity for permutation classes via the undecidability of joint embedding for hereditary graph classes (2019). Preprint available at arXiv:1903.11932

11. Bulatov, A.A.: A dichotomy theorem for nonuniform CSPs. In: 58th IEEE Annual Symposium on Foundations of Computer Science, FOCS 2017, Berkeley, CA, USA, 15-17 October 2017, pp. 319-330 (2017)

12. Cherlin, G., Shelah, S., Shi, N.: Universal graphs with forbidden subgraphs and algebraic closure. Adv. Appl. Math. 22, 454-491 (1999)

13. Courcelle, B., Engelfriet, J.: Graph Structure and Monadic Second-Order Logic: A Language-Theoretic Approach. Cambridge University Press, New York (2012)

14. Fagin, R.: Generalized first-order spectra and polynomial-time recognizable sets. Complex. Comput. 7, 43-73 (1974) 
15. Feder, T., Vardi, M.Y.: The computational structure of monotone monadic SNP and constraint satisfaction: a study through Datalog and group theory. SIAM J. Comput. 28, 57-104 (1999)

16. Feder, T., Vardi, M.Y.: Homomorphism closed vs. existential positive. In: Proceedings of the Symposium on Logic in Computer Science (LICS), pp. 311-320 (2003)

17. Foniok, J., Nešetřil, J.: Generalised dualities and maximal finite antichains in the homomorphism order of relational structures. Eur. J. Combin. 29(4), 881-899 (2008)

18. Garey, M., Johnson, D.: A Guide to NP-Completeness. CSLI Press, Stanford (1978)

19. Grohe, M.: The quest for a logic capturing PTIME. In: Proceedings of ACM/IEEE Symposium on Logic in Computer Science (LICS), pp. 267-271 (2008)

20. Gurevich, Y.: Toward logic tailored for computational complexity. In: Börger, E., Oberschelp, W., Richter, M.M., Schinzel, B., Thomas, W. (eds.) Computation and Proof Theory. LNM, vol. 1104, pp. 175-216. Springer, Heidelberg (1984). https:// doi.org/10.1007/BFb0099486

21. Hodges, W.: Model Theory. Cambridge University Press, Cambridge (1993)

22. Hubička, J., Nešetřil, J.: Homomorphism and embedding universal structures for restricted classes. Multiple-Valued Logic Soft Comput. 27(2-3), 229-253 (2016). arXiv:0909.4939

23. Kun, G.: Constraints, MMSNP, and expander relational structures. Combinatorica 33(3), 335-347 (2013). https://doi.org/10.1007/s00493-013-2405-4

24. Ladner, R.E.: On the structure of polynomial time reducibility. J. ACM 22(1), 155-171 (1975)

25. Madelaine, F.: Universal structures and the logic of forbidden patterns. In: Ésik, Z. (ed.) CSL 2006. LNCS, vol. 4207, pp. 471-485. Springer, Heidelberg (2006). https://doi.org/10.1007/11874683_31

26. Zhuk, D.: A proof of CSP dichotomy conjecture. In: 58th IEEE Annual Symposium on Foundations of Computer Science, FOCS 2017, Berkeley, CA, USA, 15-17 October 2017, pp. 331-342 (2017). https://arxiv.org/abs/1704.01914 